\title{
Micromorfología Y ESTRUCTURA de LA CUBIERTA SEMinal de CUATRO ESPECIES DE LOASACEAE JUSS. PRESENTES EN VENEZUELA
}

\author{
Eliana Noguera ${ }^{1} \&$ Damelis Jáuregui ${ }^{2}$
}

\begin{abstract}
Resumo
(Micromorfología y estructura de la cubierta seminal de cuatro especies de Loasaceae Juss. presentes en Venezuela) Se realizó el estudio morfológico y anatómico de la cubierta seminal de Gronovia scandens L., Klaprothia fasciculata (Presl.) Poston \& Nowicke, Mentzelia aspera L., y Nasa triphylla (Juss.) Weigend, especies pertenecientes a las Loasaceae. El estudio se llevó a cabo con material fresco y material herborizado. La caracterización morfológica se realizó bajo microscopio estereoscópico. Para la caracterización histológica se obtuvieron secciones transversales y longitudinales las cuales se estudiaron bajo un microscopio óptico, adicionalmente serealizaron pruebas histoquímicas. La micromorfología de la cubierta seminal fue observada en un microscopio electrónico de barrido. Se encontraron diferencias en el tamaño, color y forma de las semillas, con las cuales se puede diferenciar fácilmente cada una de las especies. En el estudio de la microescultura y la anatomía de la cubierta seminal también se observaron rasgos específicos que permiten diferenciar las cuatro especies estudiadas.
\end{abstract}

Palavras-chave: Loasaceae, cubierta seminal.

\section{Abstract}

(Seed coat micromorphology and structure of four species of Loasaceae Juss. present in Venezuela) Was carried out the morphological and anatomical study of the seed coat of Gronovia scandens L., Klaprothia fasciculata (Presl.) Poston \& Nowicke, Mentzelia aspera L., and Nasa triphylla (Juss.) Weigend, species belonging to the Loasaceae. The study was carried out with fresh material fresh and herbarium material. The characterization morphological was carried out low microscope estereoscópico. For the characterization histological transverse and longitudinal sections those were obtained which were studied under an light microscope, additionally they were carried out histochemical assays. The micromorphology of the seed coat was observed in a scanninig electronic microscope. They were differences in the size, color and it forms of the seeds, with which it can differ each one of the species easily. In the study of the microsculpturing and the anatomy of the seed coat specific features were also observed that allow to differentiate the four studied species.

Key-words: Loasaceae, seed coat.

\section{IINTRODUCCION}

Loasaceae, se distingue de otras familias relacionadas, por sus tricomas de forma multicelular y usualmente urticantes, por los variados tipos de estaminodios, por los pétalos frecuentemente cóncavos y las cápsulas valvadas, espiraladas (Lawrence 1989). Esta familia incluye aproximadamente 20 géneros y 325 especies distribuidas principalmente a lo largo de América, y solo dos géneros fuera del continente, el género Kissenia con dos especies en el sur oeste de Africa, sur de Arabia y Somalia, y el género Plakothira con tres especies en las Islas Marquesas de Polinesia (Dandy 1926; Weigend 2002). En Venezuela, la familia está representada por cuatro géneros (Gronovia, Klaprothia, Mentzelia y Nasa) y 11 especies, con distribución principalmente al norte y en la zona andina. En la actualidad luego de reevaluaciones realizadas por Weigend (1997) para el género Nasa, las especies del género Loasa presentes en Venezuela se incluyeron dentro del género Nasa.

De acuerdo a la clasificación propuesta por Weigend (1997), Loasaceae está conformada

Artigo recebido em 03/2004. Aceito para publicação em 06/2005.

${ }^{1}$ Postgrado en Botánica Agrícola, Facultad de Agronomía, Universidad Central de Venezuela, Prolongación Av. 19 de Abril, Zona Universitaria, apartado postal 4579, Estado Aragua, Maracay, 2101.

${ }^{2}$ Laboratorio de Anatomía Vegetal "Prof. Antonio Fernández" Instituto de Botánica Agrícola, Facultad de Agronomía, Universidad Central de Venezuela, Prolongación Av. 19 de Abril, Zona Universitaria, Vía El Limón, apartado postal 4579, Estado Aragua, Maracay, 2101. eliananoguera@yahoo.com 
por cuatro subfamilias: Gronovioideae, Loasoideae, Mentzelioideae y Petalonychoideae. En el presente trabajo se estudiarán las especies Gronovia scandens (Gronovioideae), Klaprothia fasciculata (Loasoideae), Mentzelia aspera (Mentzelioideae) y Nasa triphylla (Loasoideae). En Venezuela no se han realizado investigaciones con relación a la morfología y/o la anatomía de las semillas de las especies de la familia Loasaceae; sin embargo, a nivel mundial existen trabajos sobre la semilla, pero basados exclusivamente en la cubierta seminal; en tales estudios se ha demostrado que la morfología de la semilla, en este grupo, tiene importancia taxonómica (Hill 1976; Poston \& Shetler 1984; Hufford 1988). Además de su importancia reproductiva y taxonómica, las semillas de las especies de Loasaceae, tienen importancia medicinal, ya que estudios químicos has demostrado que especies pertenecientes a las subfamilias Loasoideae y Mentzelioideae presentan iridoides, (Kooiman 1974; Søren, et al. 1981; Müller et al. 1999); estos compuestos están siendo empleados farmacológicamente para sintetizar ingredientes activos promisorios en el tratamiento del cáncer y del HIV (Mølgard et al. 2001). Además de esto, exploradores y botánicos reportaron que semillas del género Mentzelia fueron importantes en la dieta de los aborígenes americanos nativos en el noroeste, centro y suroeste de los Estados Unidos (Hill 1976).

Dada la importancia citada y sumado al hecho de que han sido poco estudiadas las semillas de las especies Gronovia scandens, Klaprothia fasciculata, Mentzelia aspera, y Nasa triphylla, se justifica emprender este estudio, con el fin de conocer en detalle la anatomía de las mismas y detectar caracteres con posible valor taxonómico para la familia Loasaceae.

\section{Materiales y Métodos}

Se trabajó principalmente con material de los herbarios (MY) y MERF); las colecciones utilizadas fueron las siguientes: Gronovia scandens P. Montaldo 3648 (MY); Klaprothia fasciculata L.R.T. \& J.A.D. 11798 (MERF); Mentzelia aspera L. Cárdenas 1941 (MY) y Nasa triphylla C.E. Bénitez 1737 (MY). También se usó material de colecciones propias.

Para estudiar las características morfológicas de las semillas de cada especie se utilizó material fijado en FAA y material de herbario. Para determinar el tamaño de las semillas se midió el largo y el ancho de las mismas con papel milimetrado, bajo el microscopio estereoscópico. La descripción de la forma de las semillas y la textura de sus superficies se realizó siguiendo la terminología propuesta por Lindley (1951). El color de las semillas se determinó con la carta de colores Methuen Handbook of Colour de Kornerup \& Wanscher (1978).

Para la realización del estudio histológico de cada una de las especies, se extrajeron manualmente, las semillas de los frutos maduros y se seleccionaron tres semillas por especie, provenientes de diferentes colecciones. En el caso de G. scandens las semillas no fueron extraídas de los frutos, debido a lo delicada de la cubierta seminal de las mismas y a su difícil separación del fruto. Una vez seleccionadas las semillas se hidrataron durante 24 horas en agua destilada, luego se colocaron en agua-glicerina (1:3) durante 72 horas. Posteriormente se hirvieron por 10 minutos en agua destilada y fueron colocadas en etanol $50 \%$ durante tres horas. Seguido se realizó la deshidratación de las muestras con una serie ascendente de alcohol butílico terciario (TBA), luego seinfiltró eincluyó en paraplast (Johansen 1940). Después de incluido el material; se procedió a seccionarlo entre $7-10 \mu \mathrm{m}$ en el micrótomo de rotación. Las secciones se tiñeron con azul de toluidina acuosa al 0,05\%, sin desparafinar previamente (Sakai, 1973) y se montaron en bálsamo de Canadá, obteniendo así láminas permanentes. Todas las láminas preparadas fueron observadas en un microscopio óptico marca Leitz, modelo HMLUX3, realizando descripciones cualitativas. 
Para efectuar las pruebas histoquímicas se utilizaron láminas con secciones sin teñir. Las secciones se cubrieron con los reactivos correspondientes para cada prueba. Para identificar lignina se utilizó floroglucinol al $1 \%$ en etanol al $96 \%$, para ello se adicionó una gota de floroglucinol sobre la sección y luego se le adicionó ácido clorhídrico, se detectó la presencia de este compuesto por la aparición de una coloración roja (Roth 1964). En el caso de almidón se usó yodo yoduro de potasio, la molécula de este componente al penetrar en el almidón lo colorea de violeta a negro (Roth 1964). Se detectó la presencia de lípidos con Sudan IV, preparando este último con etanol al $80 \%$, la presencia de sustancias grasas se detecta por la aparición de un color naranja (Johansen 1940).

Para el estudio de la micromorfología de la cubierta seminal, se tomaron de muestras de frutos maduros y de herbario, dos semillas por cada especie. Una vez seleccionadas las semillas fueron metalizadas con una capa de 15-35 nm de oro-paladio en un metalizador BalTec SCB 050. Posteriormente fueron observadas y fotografiadas en un microscopio electrónico de barrido Hitachi S-2300.

\section{Resultados}

Las semillas de las cuatro especies estudiadas, presentan rasgos diferentes en cuanto a su morfología y anatomía, que permiten su fácil reconocimiento.

En cuanto a las características morfológicas de las semillas estudiadas se pueden señalar que el tamaño de las semillas es variable, sin embargo, tienden a ser pequeñas; las semillas de mayor tamaño son las de G. scandens de $3 \mathrm{~mm}$ de largo $\times 1,7$ $\mathrm{mm}$ de ancho y las de menor tamaño son las de $K$. fasciculata de $0,8 \mathrm{~mm}$ de largo $\times 0,5$ $\mathrm{mm}$ de ancho, con valores intermedios $N$. triphylla $1-1,5 \mathrm{~mm}$ de largo $\times 0,5-0,8 \mathrm{~mm}$ de ancho y M. aspera de 2,5-2,8 $\mathrm{mm}$ de largo $\mathrm{x}$ $1,2-1,4 \mathrm{~mm}$ de ancho, es importante destacar que en el caso de G. scandens solo se presenta una semilla por fruto, a diferencia de las otras especies donde se presentan varias semillas por fruto; en este último caso, se observó en ocasiones, ligeras variaciones de la forma entre las semillas de un mismo fruto. Con relación a la forma en $G$. scandens y $N$. triphylla las semillas son ovoides, en $K$. fasciculata son de forma elipsoidal y en $M$. aspera son piriformes. En cuanto al color G. scandens se diferencia del resto por su tono verde grisáceo y se observa la dominancia del color marrón, en $K$. fasciculata las semillas son marrón claro, en $M$. aspera son marrón y en $N$. triphylla son marrón oscuro. La forma de las semillas, aunada a cualquiera de las otras dos características permite diferenciar las cuatro especies; a pesar de que las semillas de $G$. scandens y $N$. triphylla son ovoides.

En cuanto a la micromorfología de las semillas, destacan las siguientes características para cada una de las especies: En las semillas de G. scandens se observan suaves depresiones irregulares (Fig. 1 y 2). En el caso de las semillas de $K$. fasciculata la superficie de la cubierta seminal se distingue por presentar células alargadas longitudinalmente, con estriaciones perpendiculares al eje mayor de las mismas y células papilosas distribuidas al azar (Fig. 3) asimismo, se debe destacar que en uno de los extremos de la semilla este patrón cambia, observándose las células con la superficie ligeramente verrucosa (Fig. 4). Con relación a la cubierta seminal de $M$. aspera, está presenta células alargadas dispuestas en hileras, las cuales a su vez presentan estriaciones perpendiculares, que terminan en forma tuberculada (Fig. 6) principalmente hacia los extremos de las semillas (Fig. 5). Y en las semillas de $N$. triphylla la superficie de la cubierta seminal es reticulada, con células poligonales, de diferentes tamaños y contornos ligeramente ondulados (Figs. 7 y 8 ).

En cuanto a los caracteres estructurales en las semillas de G. scandens la testa está conformada por dos capas (Fig. 9). La primera capa de células parenquimatosas, isodiamétricas, relativamente grandes con las paredes periclinales externas ligeramente 

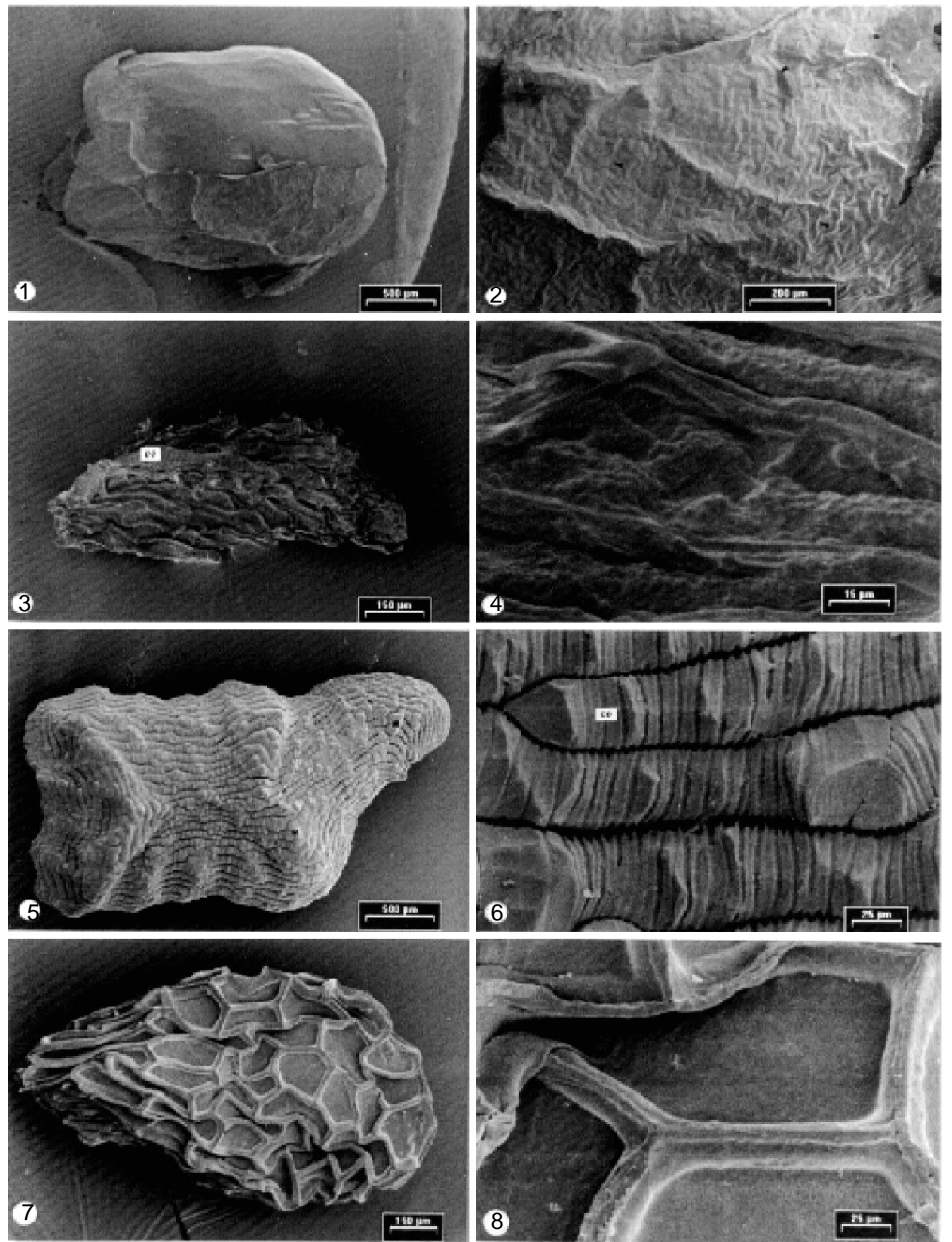

Micrografías al MEB de semillas de cuatro especies de Loasaceae. 1- Vista general de G. scandens. 2- Detalle de la cubierta seminal de G. scandens nótese suaves depresiones irregulares (indicadas con flechas). 3- Vista general de $K$. fasciculata, obsérvense células alargadas longitudinalmente con estriaciones perpendiculares (ce) células papilosas distribuidas al azar (indicadas con flecha). 4- Detalle de la cubierta seminal de K. fasciculata (cp), nótese extremo de la semilla con superficie ligeramente verrucosa. 5- Vista general de $M$. aspera, nótese células papilosas (indicadas con asterisco *). 6- Detalle de la cubierta seminal de M. aspera, obsérvense las células alargadas con estriaciones perpendiculares (ce). 7- Vista general de N. triphylla, nótese la superficie reticulada. 8) Detalle de la cubierta seminal de N. triphylla. 
convexas y las paredes anticlinales rectas. La segunda capa de células parenquimatosas relativamente pequeñas, de diferentes tamaños, de menor espesor que las células de la primera capa. En estas capas no se observó reacción a las pruebas histoquímicas realizadas (Tabla 1). En las semillas de $K$. fasciculata: la testa está constituida por una capa de células con paredes y cutícula engrosadas, donde unas paredes periclinales externas son rectas y otras convexas, presentando estas últimas aspecto papiloso (Fig. 10), y contenido citoplasmático denso. Luego se presentan de varias capas de células largas, con su eje mayor paralelo a la superficie y con engrosamientos reticulados de la pared, presentando un patrón de organización distinto al de la pared de las células de la capa externa (Fig. 11). Las paredes de estas últimas capas reaccionaron positivamente a la prueba de lípidos (Tabla 1). En cuanto a las semillas de $M$. aspera la testa está conformada por una capa muy delgada de células con contornos irregulares (Fig. 12). Seguido se observa una capa de células con las paredes periclinales externas convexas y gruesas, las paredes anticlinales con forma de omega invertida, y contenido citoplasmático denso; estas células reaccionaron positivamente a la pruebade almidón (Tabla 1) y en las paredes periclinales internas de las mismas se detectó la presencia de lípidos. Inmediatamente, se observan 2-3 capas de tejido parenquimático, donde las células tienen paredes delgadas, contenido citoplasmático denso y núcleos grandes (Fig. 12). Y para las semillas de $N$. triphylla se observó que la cubierta seminal está constituida por una capa de células de formas y tamaños diferentes con paredes ligeramente en grosadas y contenido citoplasmático relativamente denso. Las paredes periclinales externas son irregulares y las paredes anticlinales se observan rectas o curvas. Lo irregular de la forma de las células de esta capa, hace que el margen no sea homogéneo (Fig. 13). Las paredes anticlinales de esta capa reaccionaron levemente a la prueba de lípidos (Tabla 1) y en algunas células se observó reacción a la prueba de almidón. Luego de esta capa siguen 2-4 capas de células parenquimatosas de menor tamaño que las de la capa anterior, con paredes relativamente gruesas y contenido citoplasmático denso (Fig. 13), en ellas se detectó la presencia de almidón (Tabla 1).

Como se observa en la Tabla 1, en ninguna de las semillas estudiadas se detectó la presencia de lignina y se podría decir que la presencia de lípidos fue común a las especies con excepción de G. scandens.

\section{Discusión}

Las semillas de las cuatro especies estudiadas mostraron características particulares. A nivel macroscópico se pueden diferenciar fácilmente las semillas por su tamaño, forma y color. El tamaño registrado para G. scandens, $K$. fasciculata, M. aspera y $N$. triphylla coincide con lo señalado por Avendaño (1999) para estas especies.

Con relación al tamaño de las semillas Lindorf et al. (1991) señalaron que las semillas pequeñas están, por lo general, presentes en la mayoría de las plantas herbáceas; lo cual coincide con las especies aquí estudiadas, ya que estas son plantas herbáceas y presentan semillas pequeñas.

Tabla 1- Reacción ante las pruebas histoquímicas realizadas en la cubierta seminal de cuatro especies de Loasaceae, presentes en Venezuela.

\begin{tabular}{lccc}
\hline Especie & Almidón & Lípidos & Lignina \\
\hline Gronovia scandens & - & - & - \\
Klaprothia fasciculata & - & + & - \\
Mentzelia aspera & + & + & - \\
Nasa triphylla & + & + & - \\
\hline
\end{tabular}

Presencia (+) / Ausencia (-). 

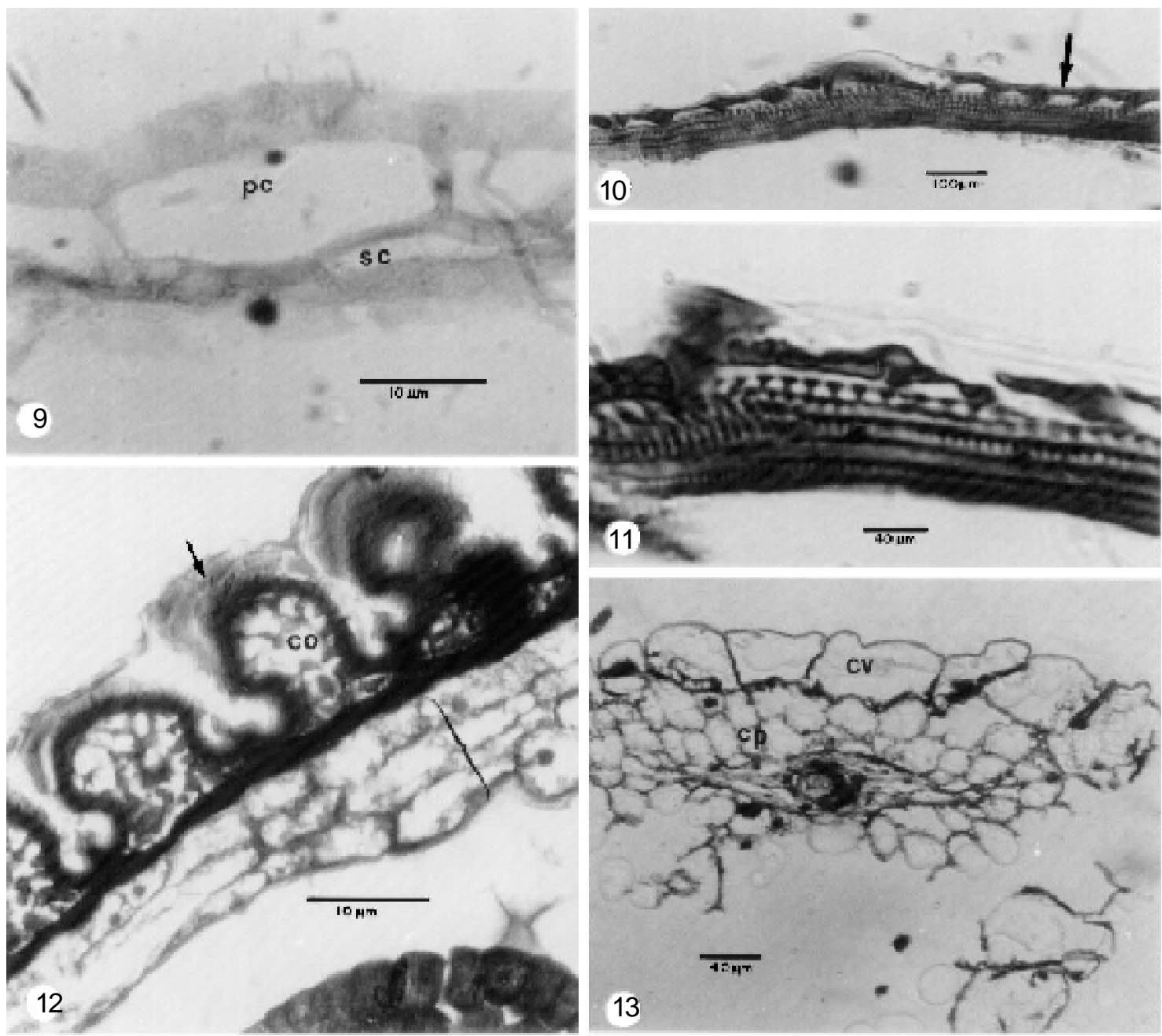

Secciones transversales de la cubierta seminal de cuatro especies de Loasaceae. 9- G. scandens, primera capa (pc) y segunda capa (sc). 10- K. fasciculata, capa de paredes gruesas (indicadas con flecha). 11- K. fasciculata células con engrosamientos reticulados en la pared (ce). 12- M. aspera células de contornos irregulares (indicados con flecha), células con forma de omega (co), células parenquimáticas de paredes delgadas (indicado con llave). 13- N. triphylla células variables en forma y tamaño (cv) células parenquimáticas pequeñas (cp).

Lindorf et al. (1991) y Werker (1997) indicaron que la disminución en el número de semillas por fruto conduce probablemente a un incremento en el tamaño de las mismas, relación esta, que se evidencia en las semillas de G. scandens. Sin embargo, al comparar las semillas de K. fasciculata y N. triphylla, no se evidencia esta relación, entre el número de semillas y su tamaño, ya que $N$. triphylla presenta mayor número de semillas por fruto y semillas de mayor tamaño que las de $K$. fasciculata.

Los resultados referentes a la forma de las semillas difieren de lo señalado por
Avendaño (1999) quien considera que las semillas de $K$. fasciculata son oval-obovoides y las de $M$. aspera son de forma irregular. Según Werker (1997) la forma de la semilla está determinada genéticamente, pero es finalmente moldeada por el espacio disponible dentro del fruto, y además indicó que la forma puede jugar un papel crucial en su dispersión.

Con relación al color Werker (1997) mencionó que la mayoría de las semillas maduras son de varios matices de marrón a negro, tal como se observó en las semillas de $K$. fasciculata, M. aspera y $N$. triphylla, y que menos comúnmente son de color gris, tal 
como se observó en G. scandens. De igual forma señaló Werker (1997) que el color de las semillas, similar al del suelo donde crecen puede ayudar en el camuflaje de las semillas para protegerlas de los depredadores. Es importante destacar que para la determinación del color de la semilla, esta debe estar madura, ya que el color puede ser variable dependiendo de su estado de desarrollo.

También la micromorfología y el arreglo histológico de la cubierta seminal aportan diferencias para la identificación de los taxa estudiados.

En cuanto a la micromorfología se puede señalar que la ornamentación de la cubierta seminal de células alargadas con estriaciones, observada en $M$. aspera coincide con la presentada por Thompson \& Powell (1981), para esta misma especie; y con la hallada por Hill (1976) para las especies M. incisa, M. floridana y M. texana. Siendo estas especies y $M$. aspera miembros de la Sección Eumentzelia, lo cual posiblemente indica que la ornamentación de la superficie de la cubierta seminal en este género, tiene importancia taxonómica.

La escultura reticulada, con contornos ondulados observada en $N$. triphylla, coincide con lo señalado por Hufford (1988) para las semillas de las especies Loasa bergii, L. incana y $L$. rudis (estas especies actualmente son consideradas dentro del género Nasa). Ambos resultados hacen presumir que el patrón reticulado tiende a ser común entre las especies de este género. Sin embargo, para la determinación específica, parece tener importancia la ornamentación de la superficie de la pared periclinal externa, o lo que según Barthlott (1981) es la escultura secundaria.

Poston \& Nowicke (1990) consideraron para la reevaluación de los géneros Klaprothia y Sclerothrix, la ornamentación de la cubierta seminal como carácter de importancia taxonómica para su identificación. Estos autores señalaron que la cubierta seminal de $K$. fasciculata es reticulada, con estriaciones perpendiculares al eje longitudinal de la semilla; coincidiendo sus resultados con lo señalado en este estudio para dicha especie.

Corner (1976) señaló para las semillas de la familia Loasaceae, que éstas provienen de óvulos unitegumentados y la testa no multiplicativa, lo cual podría explicar lo delgada de la testa en cada una de las semillas estudiadas.

De todas las semillas estudiadas las de G. scandens presentaron la testa con la estructura mas sencilla, lo cual podría deberse en parte, a que el fruto le proporciona protección al embrión; en relación a esto Lindorf et al. (1991) mencionaron que en los frutos monospermos la protección del embrión es realizada básicamente por las paredes del fruto, ya que la cubierta seminal se presenta muy poco desarrollada. Adicionalmente, señalaron que en estos casos lo difícil de la separación de la cubierta seminal del pericarpio, hace que sea conveniente tratar el conjunto como un todo indiviso, situación ésta que se presentó en las semillas de G. scandens. Debido a está condición, es posible que en las semillas de G. scandens, el fruto sea también su medio de dispersión.

Lindorf et al. (1991) señalaron que en algunas semillas la barrera más importante a la impermeabilidad no parece ser la cutícula sino, el arreglo compacto que ofrecen algunas capas celulares, ocurriendo quizás está situación en $K$. fasciculata donde claramente se observa la disposición entramada de las células de la segunda y tercera capa.

Con relación a las pruebas histoquímicas en la literatura no se encontraron trabajos que señalen datos sobre la composición química de la testa de las especies de la familia Loasaceae.

La presencia de lípidos observada en las semillas (Tabla 1) podría actuar como mecanismo de protección y regulador de la permeabilidad. La presencia de almidón puede servir como fuente de reserva para el crecimiento del embrión.

Es evidente que en estas cuatro especies confluyen diferentes estrategias para la protección del embrión. 


\section{Conclusión}

En conclusión se puede señalar que los resultados obtenidos coinciden y demuestran lo señalado por Poston \& Shetler (1984), y Hufford (1988) quienes refieren la importancia taxonómica de la semilla en la familia Loasaceae, y que tal como lo señaló Werker (1997) la anatomía de la cubierta seminal puede servir como una ayuda en la identificación y taxonomía a nivel específico. Por consiguiente las cuatro especies estudiadas pueden ser fácilmente identificadas o separadas por medio de las características morfológicas, micromorfológicas y estructurales de la cubierta seminal.

\section{Agradecimientos}

Las autoras desean expresar su agradecimiento a la Técnico Norbelis de Benítez del Laboratorio de Anatomía Vegetal "Prof. Antonio Fernández" de la Facultad de Agronomía de la Universidad Central de Venezuela. Al Prof. Ulises Castejón coordinador del centro de microscopía electrónica en el Centro de investigación y análisis docente asistencial del Núcleo Aragua de la Universidad de Carabobo (CIADANA). Al personal del Centro de Microscopia Electrónica de la Facultad de Agronomía de la UCV (CenMEFA).

\section{Referencias Bibliografiacas}

Avendaño, S. 1999. Loasaceae. In: Flora de Veracruz. Instituto de Ecología. México. Fascículo 110.27p.

Barthlott, W. 1981. Epidermal and seed surface characters of plants. Systematic aplicability and some evolutionary aspects. Nordic Journal of Botany 1: 345-355.

Corner, E. 1976. The seeds of dicotyledons. Vol. 1. Cambridge University Press. 311p.

Dandy, J. 1926. Notes on Kissenia and the geographical distribution of the Loasaceae. Bulletin of Miscellaneous Information 4: 174-180.

Hill, R. 1976. Taxonomic and phylogenetic significance of seed coat microsculpturing in Mentzelia (Loasaceae) in Wyoming and adjacent western states. Brittonia 28: 86-112.

Hufford, L. 1988. Seed morphology of Eucnide and other Loasaceae. Systematic Botany 13(1): 154-167.

Johansen, D. 1940. Plant microtehcnique. New York, McGraw-Hill. 523p.

Kooiman, P. 1974. Iridoid glycosides in the Loasaceae and taxonomic position of the family. Acta Botanica Neerlandica 23(56): 677-679.

Kornerup, A. \& Wanscher, J. H. 1978. Methuen Handbook of Colour. Third edition. Londres.

Lawrence, G. 1989. Taxonomy of vascular plants. New York, Macmillan Publishing. $823 p$.

Lindley, J. 1951. Glosología o de los términos usados en Botánica. Instituto Miguel Lillo de la Univesidad Nacional de Tucumán . Tucumán 123p.

Lindorf, H.; Parisca, L. \& Rodríguez, P. 1991. Botánica. Clasificación, Estructura y Reproducción. 2ed. Caracas, Universidad Central de Venezuela. Ediciones de la Biblioteca. 584p.

Mølgard, P.; Itenov, K.; Nyman, U.; Rønsted, N. \& Sandager, T. Special chemicals and pharmaceuticals from plants. [en línea]. Royal Danish School of Pharmacy web page. 2001, http://www.dfh.dk/instm/ forskning/pharmacognosy/projekter.htm. [consulta18 de marzo 2003].

Müller, A. A.; Kuffer, J. K.; Dietl, K. G.; Reiter, S. A.; Grau, J. \& Weigend, M. 1999. Iridoid glucosides_chemotaxonomic markers in Loasoideae. Phytochemistry 52: 67-78.

Poston, M. \& Shetler, S. 1984. Systematic importance of seed surface features in Caiphora Loasaceae (subfamily Loasoideae). Association of South Eastern Biologist Bulletin 31: 77.

Poston, M. \& Nowicke, J. 1990. A reevaluation of Klaprothia and 
Sclerothrix (Loasaceae: Klaprothieae). Systematic Botany 15(4): 671-678.

Roth, I. 1964. Microtecnía Vegetal. Facultad de Ciencias. Universidad Central de Venezuela. Caracas. 88p.

Sakai, W. 1973. Simple method for differential stainig of paraffin embedded plant material using toluidine blue O. Stain Technology 48: 247-249.

Søren, R. J.; Mikkelsen, C. B. \& Nielsen, B. J. 1981. Iridoid mono- and di-glycosides in Mentzelia. Phytochemistry 20: 71-83.
Thompson, H. \& Powell, A. 1981. Loasaceae of the Chiuahuan Desert Region. Phytologia 49(1): 16-32.

Weigend, M. 1997. Nasa \& the conquest of South America. Ph. D. Thesis. Munich.

Weigend, M. "Nasa and its allies". [en línea] Loasaceae web page. 2002, http://www. biologie.fuerlin.de/sysbot/weigend/ start.Html [consulta 15 de mayo 2002].

Werker, E. 1997. Seed Anatomy. Israel, The Hebrew University of Jerusalen. 424p. 
